Sitzungsberichte

der Akademie der Wissenschaften der DDR

Mathematik - Naturwissenschaften - Technik

\title{
Walter Kirsche
}

\section{Oskar Vogt \\ 1870-1959}


